\title{
Throughput and Outage Probability Analysis for Cognitive Radio-Non-Orthogonal Multiple Access in Uplink and Downlink Scenarios
}

\author{
Madan H.T. ${ }^{*}$, Prabhugoud I. Basarkod \\ School of Electronics and Communication, REVA University, Bengaluru 560064, India
}

Corresponding Author Email: madanht@reva.edu.in

https://doi.org/10.18280/mmep.070419

Received: 9 September 2020

Accepted: 15 November 2020

\section{Keywords:}

cognitive radio $(C R)$, non orthogonal multiple access (NOMA), underlay sharing, overlay sharing, primary users $(P U)$, secondary users $(S U)$

\begin{abstract}
Non orthogonal multiple access (NOMA) in cognitive radio (CR) network has been recognized as potential solution to support the simultaneous transmission of both primary and secondary users. In addition, CR-NOMA can also be used to serve multiple secondary networks in overlay cognitive radio networks. The aim of our work is to increase the secondary user's throughput without compromising in QoS requirements of the primary users. Our presented work analyses the performance of power domain NOMA in cognitive radio networks for both uplink and downlink scenarios. The primary aspect of the work is to investigate the impact of power allocation on spectrum efficiency and fairness performance of CR-NOMA. Objective function is to maximize the overall throughput under the QOS constraints of the users. We have derived closed form expressions for optimized power allocation coefficient $(\alpha)$ for CR-NOMA uplink and downlink communications. Parameters causing the channel outage, have been examined and conditions for outage probability is derived for CR-NOMA communication. Finally, we have presented the simulation results to validate the mathematical models that are developed for power allocation coefficient and outage probability.
\end{abstract}

\section{INTRODUCTION}

Next generation wireless networks need to provide the radio resources for heterogeneous types of wireless devices. In future, the scarcest resource will be frequency spectrum which can be rendered only by effective utilization of spectrum bands. With this motto, various wireless technologies have been emerged as potential solution, such as cognitive radio technology, full duplex communication, non-orthogonal multiple access (NOMA) etc.

\subsection{Background}

Cognitive radio technology supports dynamic spectrum allocation, which encourages sensing based spectrum sharing for unlicensed secondary users. Cognitive radio networks [1] can be operated in two modes: Underlay and overlay sharing modes. In the former case, both licensed (Primary) and unlicensed (secondary) users are allowed to utilize the same channel simultaneously. Wherein in the latter case, secondary users can opportunistically access the channel when incumbent user is inactive. It is important that, Spectrum handoff for secondary users will be done whenever the primary user becomes active. Various standards and protocols were also developed such as IEEE802.22, IEEE802.15b, etc. to practically implement cognitive radio approach in wireless communication. Several applications like rural broadband, wireless connectivity in borders, 4G/5G backhauls have successfully adopted cognitive radio technology for their communication. Despite this, CRN has faced few challenges and regulatory issues in the practical implementation. Thus, the modern researchers showed interest in developing the hybrid wireless technologies such as CR-NOMA, NOMA-FD, MIMO-FD etc. [2, 3]. In the recent wireless research, nonorthogonal multiple access has been extensively studied to explore its compatibility for IOT and 5G implementation. NOMA supports multiple data flow in the same orthogonal block either in power or code domain. To superimpose multiple users signal, NOMA [4] uses superposition coding (SC) at the transmitter and successive interference cancellation (SIC) at the receiver side. Presented work involves the integration of NOMA into cognitive radio networks to facilitate coexistence of primary and secondary communication and also self-coexistence of multiple secondary communications. CR-NOMA uses the benefits of both technologies, so it has been examined as one of the driving forces for next generation wireless communication. Integration of NOMA into CRN contributes to intelligent spectrum detection and decision; moreover, spectrum efficiency will be greatly enhanced. Conceptually CR-NOMA is the variant of power domain NOMA which is optimized to meet the QOS requirement of the users.

\subsection{Related works}

Cognitive radio technology [5] involves channel/spectrum sensing, decision, sharing, handoff, and many other aspects of spectrum management. Various researchers have been contributed on these aspects to improve the spectrum efficiency and to prevent the primary transmission from the harmful interference. Since our current work focuses on spectrum sharing aspect, we have extensively studied on how 
to incorporate NOMA into cognitive radio networks. Several studies $[6,7]$ have provided the evidence of performance enhancement by NOMA when coupled with existing wireless technologies like MIMO, FD, beam forming etc. Many researchers $[8,9]$ have intensively studied different variants and derivatives of non-orthogonal multiple access, their potential challenges, and Opportunities in future wireless communication. NOMA can be implemented either in power domain or code domain, Islam et al. [10] has shortlisted the scenarios suitable for their adoption. Wei et al. [11] has highlighted the significant benefits of NOMA over other orthogonal access methods. There exist a several literatures $[12,13]$ on energy efficient methods to implement power domain NOMA, most of them used Simultaneous Wireless Information and Power Transfer (SWIPT) technique. Majority of these research works emphasizes on possible ways of energy harvesting in wireless communication, rather they do not deal with the optimization of power allocation according the QOS requirements. In the survey articles [14, 15], authors have presented the different frameworks for the implementation of NOMA into CRN and their feasibility in gaining the higher spectrum efficiency. Liu et al. [16] has presented a review on power domain NOMA which supports multiple transmission at different power levels and uses SC at the base station and SIC at the user end for power domain NOMA. Also, authors have highlighted some of the impacts of power allocation on the user data rates. Conventionally, NOMA- power allocation depends upon the channel conditions, user with low channel coefficient will be allocated with more power for fairness. It is a common myth [17] that NOMA always allocates higher power levels to the poor channel users. In cognitive radio coupled NOMA (CRNOMA), power allocation strategies to be established such that QOS requirements of every user is satisfied.

\subsection{Contributions and organization}

By considering the data rate requirements of the participation networks, power allocation factor of CR-NOMA is optimized towards achieving the maximum overall throughput. The effectiveness is verified and validated by outage probability analysis for different values of channel coefficients. In this article, (a) we have proposed the feasible CR-NOMA frameworks, where NOMA can be effectively integrated with cognitive radio technology (Sec.2). (b) Impact of power allocation on performance of CR-NOMA is investigated for underlay and overlay sharing in downlink scenario (Sec.3). (c) Thus, derived the optimum value of power allocation coefficient required to meet the desired data rates (Sec. 3). (d) Further the analytical expressions are derived for outage probability conditions (Sec. 4). (e) To evaluate the mathematical expressions, simulation results are obtained for CR-NOMA and compared with traditional OMA techniques (Sec. 5).

\section{SYSTEM MODEL: COGNITIVE RADIO-NOMA COMMUNICATION}

CR-NOMA is the derivative of power domain NOMA through which simultaneous communication of primary and secondary users is made possible without causing harmful interference to primary users (Figure 1).

\subsection{Downlink scenario}

In CR-NOMA downlink scenario, the transmitter must serve multiple users belonging to different networks. The encoded information from different networks are combined to produce superposition coded signal and broadcasted to all the users in that coverage area. CR-NOMA downlink scenario can be implemented in two ways: Underlay sharing and Interweave sharing. In underlay CR-NOMA scenario, the radio resources are simultaneously exploited by primary and secondary networks. But the presence of secondary communication shouldn't affect the QOS requirements of primary communication. Signal from primary and secondary base stations are superposition coded and transmitted from the same transceiver. Prerequisite for this method is- primary and secondary base stations should coordinate with single transmitter. In interweave CR-NOMA scenario, the spectrum sensing $[18,19]$ is employed to know whether spectrum is being used by primary user or not. Generally, energy detection is used for single node sensing and cooperative sensing for making final decision about the whitespace availability. Transmission is permitted only if the primary user is idle: spectrum whitespaces are identified. Here, multiple secondary users can participate in transmission in coordination with fusion Centre. This method is more suitable for wireless sensor network scenarios, where multiple small-scale networks participate in spectrum access. In interweave spectrum sharing, sensing and handoff play very important role in protecting primary users from the secondary interference. In the process of SIC decoding (at the user side), Primary user will first decode the partner user's signal and its own signal is recovered by subtracting decoded signal from the received SC signal (Figure 2). In Interweave sharing approach, user with high priority (or QoS) can be treated as primary signal.

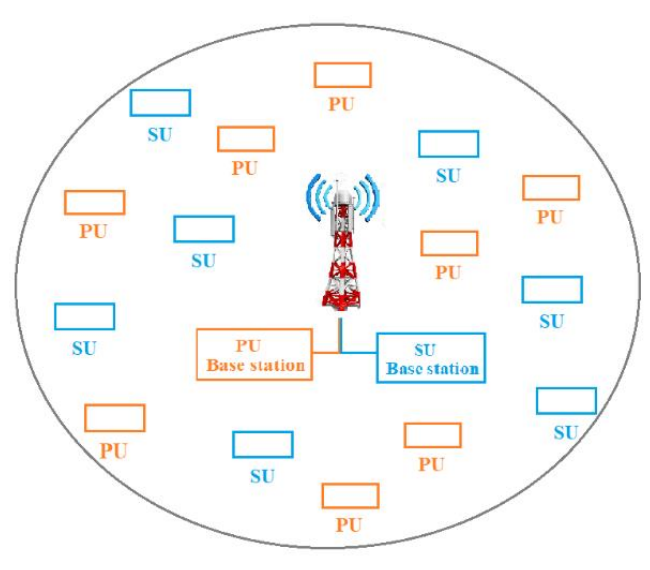

Figure 1. Cognitive radio-NOMA system model

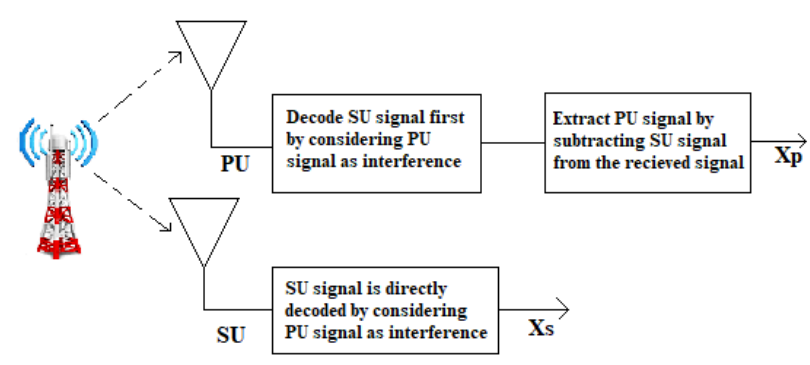

Figure 2. Decoding scheme for downlink scenario 


\subsection{Uplink scenario}

In uplink CR-NOMA scenario, multiple users simultaneously transmit their signal towards the base station in the same orthogonal block (same frequency and at the same time). There is not much difference between NOMA and CRNOMA in uplink scenario.

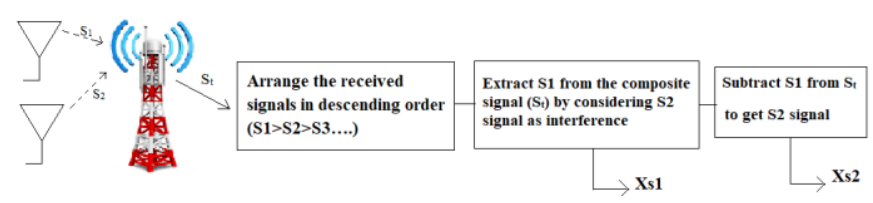

Figure 3. Decoding scheme for up-link scenario

In uplink, the power allocation can be done in either full power mode or controlled power mode. In CR-NOMA, secondary users are operated in power-controlled mode and primary users in full power allocated mode. The receiver must have SIC capability to decode signals received from multiple users: the process is as follows (Figure 3): Received signals are first ordered in descending list based on signal strength and then successive interference cancellation is applied to extract and decode the symbols.

Since the priority is given to primary user in power allocation, probably received PU signal strength will be more than that of SU signal. However, because of SIC, decoding of SU signal doesn't suffer from the interference of PU signal at the transceiver. Though secondary users are operated under power-controlled mode, they enjoy interference free communication in uplink scenario.

\section{POWER ALLOCATION OPTIMIZATION}

In this section, we have presented the power allocation model for cognitive radio NOMA communication. In conventional NOMA, power is allocated based on the channel state information i.e. user with poor channel condition will get more power allocated to bring fairness in data rates. But in CRNOMA, primary users are high priority users, their QOS needs to be strictly met. So, power allocation needs to be done depending on the QOS requirements. In this article, we have presented a power allocation algorithm which is primarily based on QOS constraints and also considers channel gain to yield maximum overall throughout. Figure 2 shows the downlink scenario, where users belonging to different networks receive the same composite SC signal.

The transmitted super position coded signal can be modelled as:

$$
x=\sqrt{\alpha \rho} x_{p}+\sqrt{\bar{\alpha} \rho} x_{s}
$$

The received signal at the user end will be:

$$
y_{i}=h_{i}\left(\sqrt{\alpha \rho} x_{p}+\sqrt{\bar{\alpha} \rho} x_{s}\right)+w_{i}
$$

For $\mathrm{N}=2$, user 1(high priority) first decodes the user'2 signal and then subtracts user' 2 signal from the received signal $y_{i}$. The maximum achievable rate for user1 can be calculated by Eq. (3):

$$
\mathrm{R}_{1}=\mathrm{B}_{\mathrm{D}} \log _{2}\left(1+\gamma_{1}\right)=\log _{2}\left(1+\alpha \rho \beta_{1}\right)
$$

At user 2, signal is directly decoded from the SC signal by assuming user' 1 signal as interference. Thus, the achievable data rate for user 2 is given by:

$$
R_{2}=B_{D} \log _{2}\left(1+\gamma_{2}\right)=\log _{2}\left(1+\frac{\bar{\alpha} \rho \beta_{2}}{1+\alpha \rho \beta_{2}}\right)
$$

The total throughput achievable for two users downlink scenario is:

$$
R=\log _{2}\left(1+\alpha \rho \beta_{1}\right)+\log _{2}\left(1+\frac{\bar{\alpha} \rho \beta_{2}}{1+\alpha \rho \beta_{2}}\right)
$$

\subsection{Lemma1: Underlay CR-NOMA}

In this approach, secondary users can concurrently access the primary user spectrum if interface level is not harmful. Primary user poses a QOS constraint on power allocation optimization. Thus, the concern is to provide the maximum possible bandwidth for secondary communication without compromising the PU QoS constraints.

The problem can be mathematically modelled as:

$$
\begin{gathered}
\alpha=\operatorname{argmax}\left(R_{s}\right) \\
\text { s.t } R_{p}>\overline{R_{P}}
\end{gathered}
$$

The condition evaluates to:

$$
\log _{2}\left(1+\alpha \rho \beta_{p}\right) \geq \overline{R_{P}}
$$

Here primary user is user 1 and $\overline{\mathrm{R}_{\mathrm{P}}}$ is the minimum QoS requirement of primary user. By solving Eq. (6) and considering the constraint given in Eq. (7), we get:

$$
\alpha_{\text {min }}=\frac{2^{\overline{\mathrm{R}_{\mathrm{P}}}}-1}{\rho \beta_{\mathrm{p}}} \geq \frac{\overline{\overline{\mathrm{R}}}_{\mathrm{P}}}{\rho \beta_{\mathrm{p}}} \text {, where } \overline{\overline{\mathrm{R}}}_{\mathrm{P}}=2^{\overline{\mathrm{R}_{\mathrm{P}}}}-1
$$

where, $\alpha$ is the optimized power allocation coefficient (Eq. (8)) which maximizes the secondary throughout by strictly meeting the QoS constraint of PU. Any value of $\alpha$ less than $\alpha_{\min }$ will degrade the performance of PU network.

\subsection{Lemma 2: Interweave CR-NOMA}

In this sharing framework, spectrum sensing is used to identify the White spaces. If the spectrum white spaces are identified with the probability of detection 0.9 , only then secondary communication is allowed. Spectrum handoff plays a key role in the protection of PU. Thus, the prerequisite for this method is: proper sensing and handoff scheme must be employed. In interweave sharing, multiple small-scale secondary networks will coordinate with fusion Centre and participate in the transmission. So, the power allocation must consider the QOS requirements of all participating networks. For $\mathrm{N}=2$, network with higher priority is $\mathrm{Sn} 1$ and other one is $\mathrm{Sn} 2$. The objective function of the problem statement is to maximize the total throughput by meeting the individual QoS rates.

The problem can be mathematically modelled as:

$$
\begin{aligned}
\alpha & =\operatorname{argmax}(\mathrm{R}) \\
\text { s. } \mathrm{R}_{\mathrm{S} 1} & \geq \overline{\mathrm{R}_{\mathrm{S} 1}} \& \mathrm{R}_{\mathrm{S} 2} \geq \overline{\mathrm{R}_{\mathrm{S} 2}}
\end{aligned}
$$


where, $R_{\mathrm{s} 1}$ and $\mathrm{R}_{\mathrm{s} 2}$ are the achievable throughput for secondary networks $\mathrm{Sn} 1$ and $\mathrm{S}_{\mathrm{n} 2} \cdot \overline{\mathrm{R}_{\mathrm{S} 1}}$ and $\overline{\mathrm{R}_{\mathrm{S} 2}}$ are the minimum targeted QoS rates of secondary networks $\mathrm{Sn} 1$ and $\mathrm{S}_{\mathrm{n} 2}$.

$$
\begin{gathered}
\log _{2}\left(1+\alpha \rho \beta_{\mathrm{s} 1}\right) \geq \overline{\mathrm{R}_{\mathrm{S} 1}} \\
\log _{2}\left(1+\frac{\bar{\alpha} \rho \beta_{\mathrm{s} 2}}{1+\alpha \rho \beta_{\mathrm{s} 2}}\right) \geq \overline{\mathrm{R}_{\mathrm{S} 2}}
\end{gathered}
$$

The above conditions evaluate to

$$
\frac{\overline{\overline{\mathrm{R}}}_{\mathrm{S} 1}}{\rho \beta_{\mathrm{S} 1}} \leq \alpha \leq \frac{\rho \beta_{\mathrm{S} 2}-\overline{\overline{\mathrm{R}}}_{\mathrm{S} 2}}{\rho \beta_{\mathrm{S} 2}\left(1+\overline{\overline{\mathrm{R}}}_{\mathrm{S} 2}\right)}
$$

By carefully analyzing the expressions Eq. (12) and Eq. (5), we can conclude that maximum net throughput occurs at $\alpha=\alpha_{\text {opt }}$.

$$
\alpha_{\mathrm{opt}}=\frac{\rho \beta_{\mathrm{S} 2}-\overline{\overline{\mathrm{R}}}_{\mathrm{S} 2}}{\rho \beta_{\mathrm{S} 2}\left(1+\overline{\overline{\mathrm{R}}}_{\mathrm{S} 2}\right)}
$$

\subsection{Power allocation for uplink scenario}

In uplink, Base station receives superposed signal consisting of signals from primary and secondary users. These signals are of different strength depending upon the user uplink channel conditions. Thus, uplink data rates depend upon channel gain, power allocation and decoding schemes adopted in the base station.

The received signal at the base station is:

$$
y=\sqrt{\alpha \rho} x_{p} h_{p}+\sqrt{\bar{\alpha} \rho} x_{s} h_{s}+N_{0}
$$

In underlay uplink scenario, primary users are operated in full power mode and secondary users are operated under controlled power mode. Due to this, received PU signal strength will be probably higher than that of SU. Considering the above probability in uplink decoding scheme, PU symbol is decoded first by treating SU signal as interference and SU symbol is decoded later by subtracting PU signal from the received superposed signal.

Thus, uplink data rates are:

$$
\begin{gathered}
\mathrm{R}_{\mathrm{p}}=\mathrm{B}_{\mathrm{U}} \log _{2}\left(1+\frac{\alpha \rho\left|\mathrm{h}_{\mathrm{p}}\right|^{2}}{1+\bar{\alpha} \rho\left|\mathrm{h}_{\mathrm{s}}\right|^{2}}\right) \\
\mathrm{R}_{\mathrm{s}}=\mathrm{B}_{\mathrm{U}} \log _{2}\left(1+\bar{\alpha} \rho\left|\mathrm{h}_{\mathrm{s}}\right|^{2}\right)
\end{gathered}
$$

To meet the QoS requirements of the primary user, $\left(\mathrm{R}_{\mathrm{p}}>\right.$ $\left.\overline{\mathrm{R}_{\mathrm{P}}}\right)$ :

$$
\begin{gathered}
\mathrm{B}_{\mathrm{U}} \log _{2}\left(1+\frac{\alpha \rho\left|\mathrm{h}_{\mathrm{p}}\right|^{2}}{1+\bar{\alpha} \rho\left|\mathrm{h}_{\mathrm{s}}\right|^{2}}\right) \geq \overline{\mathrm{R}_{\mathrm{P}}} \\
\alpha \rho\left|\mathrm{h}_{\mathrm{p}}\right|^{2} \geq\left(2^{\overline{\mathrm{R}_{\mathrm{P}}}}-1\right)\left(1+\bar{\alpha} \rho\left|\mathrm{h}_{s}\right|^{2}\right)
\end{gathered}
$$

To satisfy the above-mentioned requirements, the received signal strength must be greater than that of in Eq. (18), otherwise decoding schemes must be done in opposite way to provide interference free communication for PU. Thus, expressions for uplink data rates will be modified to:

$$
\begin{gathered}
\mathrm{R}_{\mathrm{p}}=\mathrm{B}_{\mathrm{U}} \log _{2}\left(1+\alpha \rho\left|\mathrm{h}_{\mathrm{p}}\right|^{2}\right) \\
\mathrm{R}_{\mathrm{s}}=\mathrm{B}_{\mathrm{U}} \log _{2}\left(1+\frac{\bar{\alpha} \rho\left|\mathrm{h}_{\mathrm{s}}\right|^{2}}{1+\bar{\alpha} \rho\left|\mathrm{h}_{\mathrm{p}}\right|^{2}}\right)
\end{gathered}
$$

In case of interweave uplink communication, users belonging to multiple secondary networks are operated under equal power mode and base station implements SIC depending upon the received signals strength (Explained in Sec. 2).

\section{SYSTEM OUTAGE PROBABILITY}

If channel condition of the user is not able to support the desired rate, then there will be outage. It is not always possible to support the desired QoS because of fading channel. To analyze the system outage, we must consider both downlink and uplink scenarios [20].

\subsection{Outage probability analysis for CR-NOMA downlink scenario}

We have taken underlay cognitive radio communication for downlink outage probability analysis. $\overline{\mathrm{R}_{\mathrm{P}}}$ and $\overline{\mathrm{R}_{\mathrm{s}}}$ are the minimum rates required for primary and secondary networks, respectively. Priority is given to PU network such that $R_{p}>\overline{R_{P}}$. The decoding order at the user level is as described in section Transmission scheme must ensure that decoding at the users is reliable.

\section{Primary user channel:}

Probability of outage corresponding to primary network user is:

$$
\mathrm{P}_{\text {outage }}=\mathrm{P}\left(\mathrm{C}_{\mathrm{p}}^{\mathrm{x}_{\mathrm{s}}}<\overline{\mathrm{R}_{\mathrm{s}}} \cup \mathrm{C}_{\mathrm{p}}^{\mathrm{x}_{\mathrm{p}}}<\overline{\mathrm{R}_{\mathrm{p}}}\right)
$$

where, $C_{p}^{x}$ is achievable capacity of the PU for decoding the signal $\mathrm{x}$.

This first condition says, outage occurs when:

$$
\mathrm{C}_{\mathrm{p}}^{\mathrm{x}_{\mathrm{s}}}=\log _{2}\left(1+\frac{\bar{\alpha} \rho \beta_{\mathrm{p}}}{1+\alpha \rho \beta_{\mathrm{p}}}\right)<\overline{\mathrm{R}_{\mathrm{s}}}
$$

Eq. (22) evaluates to the condition:

$$
\beta_{p}<\frac{\overline{\overline{\mathrm{R}}}_{\mathrm{S}}}{\rho\left(\bar{\alpha}-\alpha \overline{\overline{\mathrm{R}}}_{\mathrm{S}}\right)}
$$

The second condition that may lead to primary user outage is:

$$
\begin{gathered}
C_{p}^{x_{p}}=\log _{2}\left(1+\alpha \rho \beta_{P}\right)<\overline{R_{p}} \\
\beta_{P}<\frac{\overline{\bar{R}}_{P}}{\rho \alpha}
\end{gathered}
$$

Therefore, primary user outage occurs when the channel coefficient: 


$$
\beta_{p}<\max \left\{\frac{\overline{\overline{\mathrm{R}}}_{\mathrm{P}}}{\rho \alpha}, \frac{\overline{\overline{\mathrm{R}}}_{\mathrm{S}}}{\rho\left(\bar{\alpha}-\alpha \overline{\overline{\mathrm{R}}}_{\mathrm{S}}\right)}\right\}
$$

If we assume $|\mathrm{h}|$ as Rayleigh fading coefficient, then $\beta=\left|h_{p}\right|^{2}$ will be exponentially distributed. So, Eq. (26) leads to:

$$
\mathrm{P}_{\text {outage }}=1-\exp \left(-\frac{1}{\delta^{2}} \max \left\{\frac{\overline{\overline{\mathrm{R}}}_{\mathrm{P}}}{\rho \alpha}, \frac{\overline{\overline{\mathrm{R}}}_{\mathrm{S}}}{\rho\left(\bar{\alpha}-\alpha \overline{\overline{\mathrm{R}}}_{\mathrm{S}}\right)}\right\}\right)
$$

\section{Secondary user channel:}

Probability of outage corresponding to secondary network user is:

$$
\mathrm{P}_{\text {outage }}=\mathrm{P}\left(\mathrm{C}_{\mathrm{s}}^{\mathrm{x}_{\mathrm{s}}}<\overline{\mathrm{R}_{\mathrm{s}}}\right)
$$

The outage occurs when:

$$
\mathrm{C}_{\mathrm{s}}^{\mathrm{x}_{\mathrm{s}}}=\log _{2}\left(1+\frac{\bar{\alpha} \rho \beta_{\mathrm{s}}}{1+\alpha \rho \beta_{\mathrm{s}}}\right)<\overline{\mathrm{R}_{\mathrm{s}}}
$$

Considering $|\mathrm{h}|$ as Rayleigh fading coefficient, Outage probability for secondary network user will be:

$$
\mathrm{P}_{\text {outage }}=1-\exp \left(\frac{\overline{\overline{\mathrm{R}}}_{\mathrm{S}}}{\rho \delta^{2}\left(\bar{\alpha}-\alpha \overline{\overline{\mathrm{R}}}_{\mathrm{S}}\right)}\right)
$$

The Eq. (27) and Eq. (30) gives the individual outage probabilities for underlay downlink scenario (for $\mathrm{N}=2$ ).

\subsection{Outage probability analysis for CR-NOMA uplink scenario}

Declaring $\mathrm{Rp}$ and $\mathrm{Rs}$ as desired rates for $\mathrm{PU}$ and $\mathrm{SU}$ respectively, the system outage is defined as probability that any one or both user in outage. In uplink scenario, the received superposition signal at the base station will be:

$$
y=\sqrt{\alpha \rho} \mathrm{x}_{\mathrm{p}} \mathrm{h}_{\mathrm{p}}+\sqrt{\bar{\alpha} \rho} \mathrm{x}_{\mathrm{s}} \mathrm{h}_{s}+N_{0}
$$

As per the Eq. (31), two channel coefficients $\left|h_{p}\right|$ and $\left|h_{s}\right|$ affects the performance in uplink communication. Since we are following nonfixed NOMA in uplink scenario, outage probability depends upon which user symbol is decoded first. Considering the worst case where received primary signal strength is less than that of given by Eq. (17), secondary signal is decoded first by treating PU signal as interference. So, the primary user outage occurs when:

$$
\left(\mathrm{C}_{\text {uplink }}^{\mathrm{x}_{\mathrm{s}}}<\overline{\mathrm{R}_{\mathrm{s}}} \cup \mathrm{C}_{\text {uplink }}^{\mathrm{x}_{\mathrm{p}}}<\overline{\mathrm{R}_{\mathrm{p}}}\right)
$$

From Eq. (15) and (16)

$$
\begin{aligned}
& \mathrm{C}_{\text {uplink }}^{\mathrm{x}_{\mathrm{s}}}=\mathrm{B}_{\mathrm{U}} \log _{2}\left(1+\frac{\bar{\alpha} \rho\left|\mathrm{h}_{\mathrm{s}}\right|^{2}}{1+\alpha \rho\left|\mathrm{h}_{\mathrm{p}}\right|^{2}}\right)<\overline{\mathrm{R}_{\mathrm{s}}} \\
& \mathrm{C}_{\text {uplink }}^{\mathrm{x}_{\mathrm{p}}}=\mathrm{B}_{\mathrm{U}} \log _{2}\left(1+\alpha \rho\left|\mathrm{h}_{\mathrm{p}}\right|^{2}\right)<\overline{\mathrm{R}_{\mathrm{p}}}
\end{aligned}
$$

Eq. (33) leads to

$$
1+\frac{\bar{\alpha} \rho\left|\mathrm{h}_{\mathrm{s}}\right|^{2}}{1+\alpha \rho\left|\mathrm{h}_{\mathrm{p}}\right|^{2}}<\overline{\overline{\mathrm{R}}}_{\mathrm{S}} \text { where } \overline{\overline{\mathrm{R}}}_{\mathrm{S}}=2^{\overline{\mathrm{R}_{\mathrm{S}}}}-1
$$

Solving above expression, condition for PU outage is:

$$
\left|\mathrm{h}_{\mathrm{p}}\right|^{2}<\frac{\overline{\overline{\mathrm{R}}}_{\mathrm{S}}-\bar{\alpha} \rho\left|\mathrm{h}_{\mathrm{s}}\right|^{2}}{\alpha \rho \overline{\mathrm{R}}_{\mathrm{S}}}
$$

However, Eq. (34) leads to

$$
\left|\mathrm{h}_{\mathrm{p}}\right|^{2}<\frac{\overline{\overline{\mathrm{R}}}_{\mathrm{P}}}{\alpha \rho}
$$

Primary user outage occurs if any one of the above conditions (Eq. (36) and Eq. (37)) is met:

$$
\left|\mathrm{h}_{\mathrm{p}}\right|^{2}<\max \left\{\frac{\overline{\overline{\mathrm{R}}}_{\mathrm{p}}}{\rho \alpha}, \frac{\left(\bar{\alpha} \rho\left|\mathrm{h}_{\mathrm{s}}\right|^{2}-\overline{\overline{\mathrm{R}}}_{\mathrm{S}}\right)}{\left(\alpha \rho \overline{\mathrm{R}}_{\mathrm{S}}\right)}\right\}
$$

The maximum achievable data rate in uplink for PU is given by the Eq. (15). The minimum guaranteed data rates in uplink scenario for secondary user essentially depends upon channel condition and decoding scheme employed and is given by the Eq. (33). As per the analysis shown above, the condition for secondary user outage in uplink scenario is shown below:

$$
\left|\mathrm{h}_{\mathrm{s}}\right|^{2}<\frac{\overline{\overline{\mathrm{R}}}_{\mathrm{S}}\left(1+\alpha \rho\left|\mathrm{h}_{\mathrm{p}}\right|^{2}\right)}{\bar{\alpha} \rho}
$$

It can be clearly seen from Eq. (38) and Eq. (39) that, uplink outage probability depends upon the channel coefficients and the decoding scheme employed at the base station.

\section{RESULTS AND DISCUSSIONS}

In this section, obtained simulation results are presented to evaluate and validate our proposed mathematical models for power allocation and outage probability. The complete simulation is performed by normalizing the channel bandwidth to unity. Necessary simulation variables (Table 1) are assumed according to 802.22 standards [21] and NOMA conventions. To validate the effectiveness and adoptability of NOMA in CR communication, we have plotted the comparative results (Figure 4) of CR-NOMA with regular CROMA for different values of channel coefficients. For a given value of $\alpha$, it can be visualized that performance of CR-NOMA and CR-OMA are almost equal for uniform channel conditions. But CR-NOMA outperforms CR-OMA in heterogeneous scenario; when there are users with different channel coefficients and different QoS requirements. The simulation(Figure 4) is performed with $\alpha=0.8$ and a) |hd1 $|=|$ hd2 $\mid=0.5$ b) $\mid$ hd $1|=| 0.8$, |hd2 $\mid=0.2$.

The Figure 5(a) shows the relative changes in the user's data rates for continuous variation of power allocation coefficient. $\alpha_{\min }$ indicates the minimum power required to maintain the QOS rates of PU and thus providing maximum secondary throughput. Figure 5(b) depicts the bandwidth sharing among the users for Interweave CR- NOMA communication framework. $\alpha_{\min }, \alpha_{\max }$ shows the limits of alpha within which we can meet the requirements of both the secondary networks. The above simulations were carried out for (a) $\mathrm{Rp}=4.5$ (b) $\mathrm{R}_{\mathrm{S} 1}=3, \mathrm{R}_{\mathrm{S} 2}=1$. 
Table 1. Simulation parameters

\begin{tabular}{ccc}
\hline Sl. No & \multicolumn{2}{c}{ Simulation Parameters } \\
\hline 1. & SNR range & 0 to $25 \mathrm{~dB}$ \\
2. & Down link BD and Uplink BU & Normalized to unity \\
3. & Fading channel model & Rayleigh fading Model: $\beta=|\mathrm{h}|^{2}, \delta^{2}=\mathrm{E}\{\beta\}$ \\
4. & Power allocation coefficient $(\alpha)$ & $\alpha->0$ to 1, \\
& & Calculation shown in Sec.3, using Eq. (8) and Eq. (12) \\
Primary user -RP
\end{tabular}
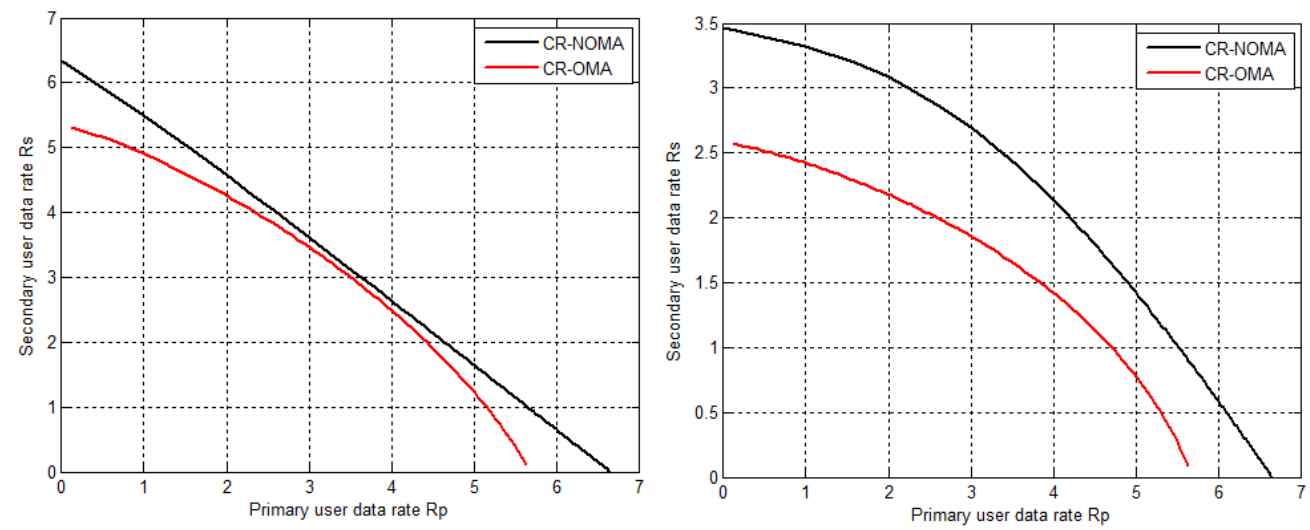

Figure 4. Throughput comparison of CR-NOMA vs CR-OMA (a) |hd1|=|hd2| (b) |hd1| > |hd2|
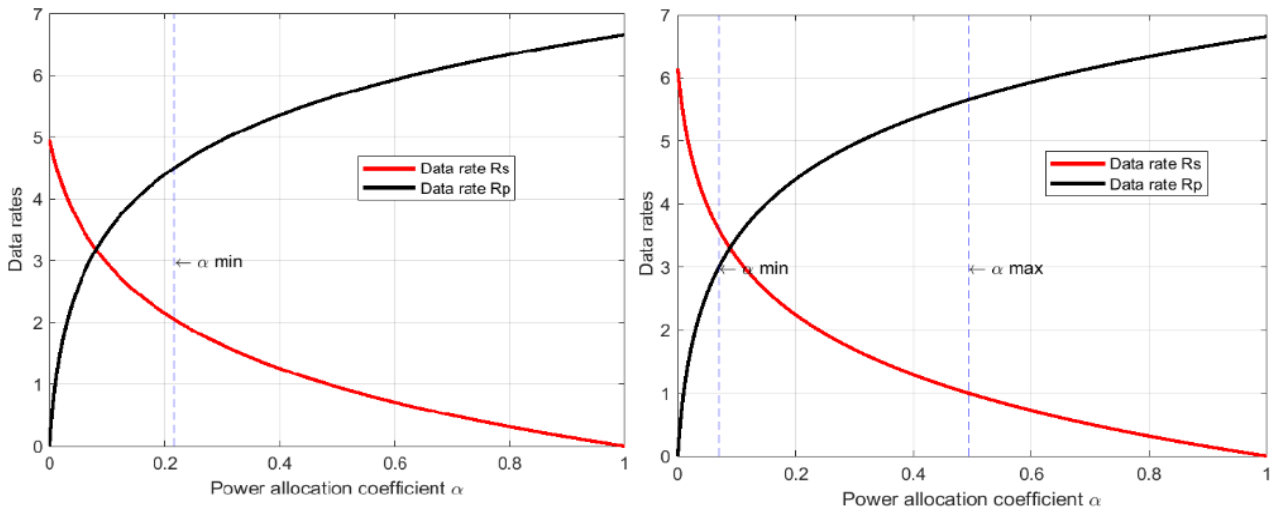

Figure 5. Data rates distribution among the user in downlink scenario (a) underlay (b) interweave sharing

The proposed algorithm outperforms conventional NOMA in cognitive radio networks in terms of overall system throughput even satisfying the QOS requirements of users of individual network. The outage probability analysis is done by considering both downlink and uplink scenarios for both ordered channels and unordered channels. In Figure 6, We have presented the outage probabilities of $\mathrm{PU}$ and $\mathrm{SU}$ for downlink conventional NOMA, where power allocation is done based solely on channel coefficients. In this case, primary user suffers more outage than the secondary users. Figure 6 shows the outage probabilities of PU and SU for optimized CR-NOMA, wherein power is allocated based on the required QoS rate and channel conditions. The above simulations were carried out by keeping fixed QoS rates and changing only power allocation coefficients. On comparison (a \&b), it can be clearly seen that outage probability of PU has been greatly reduced by adopting QoS based power allocation technique without affecting the secondary user performance.
In uplink CR communications, secondary nodes are usually operated under low power mode which reduces the throughput performance of secondary network. If both primary and secondary are operated in equal power mode, then primary network suffers more outage due to interference. Thus, uplink power allocation is optimized to increase secondary data rate simultaneously preventing primary users from outage (Figure 7). The PU outage probability is significantly reduced in power-controlled/optimized mode. It can be witnessed that system outage performance is optimal at the desired rates $\overline{\mathrm{R}_{\mathrm{P}}}<\mathrm{Rp}$ and $\overline{\mathrm{R}_{\mathrm{s}}}<\mathrm{Rs}$. As we increase the desired rates and perform the simulation, system outage probability increases drastically. For the given QoS rates, probability of outage decreases with increase in SNR, continuously up to certain value. Afterwards, SNR has very less impact on outage probability. At higher values of SNR, expected data rate and power allocation coefficient are the deciding factors. 

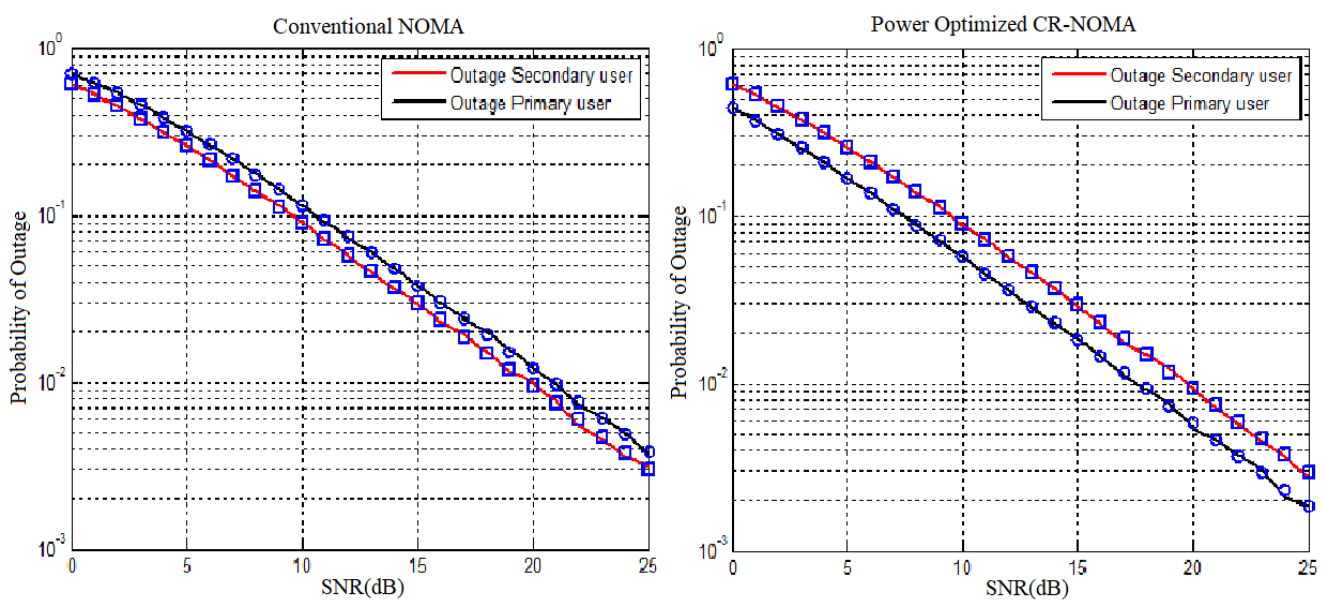

Figure 6. Downlink Outage Probability analysis: (a) Conventional CR-NOMA (b) Optimized CR-NOMA
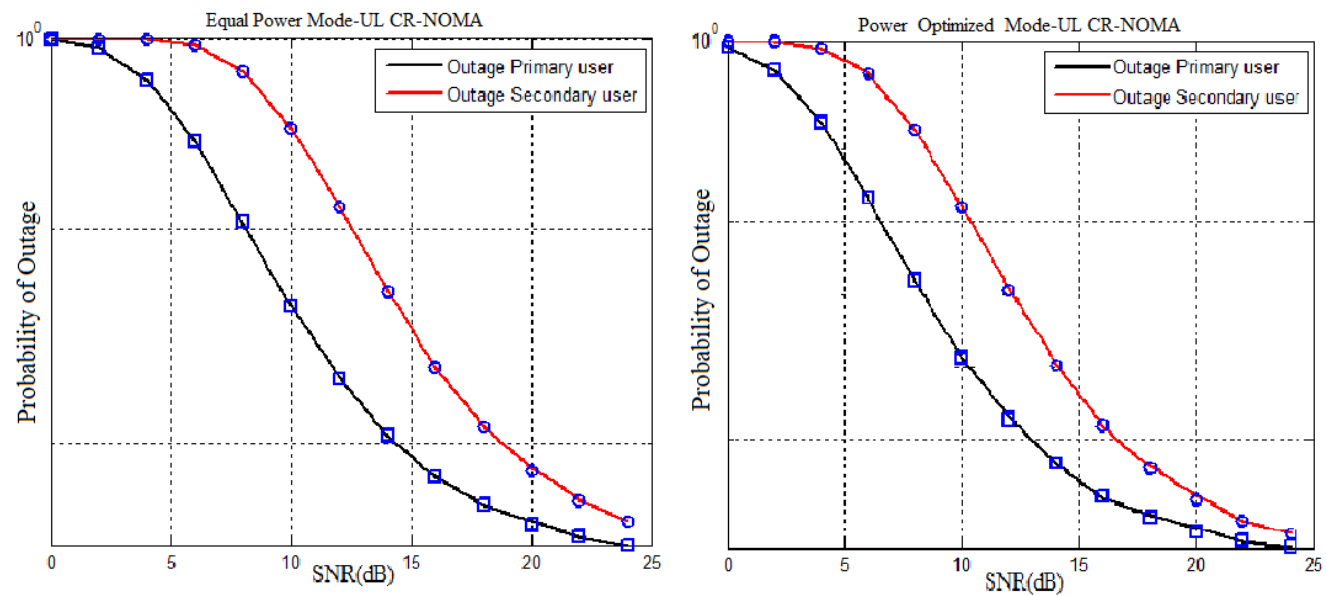

Figure 7. Uplink outage probability analysis: (a) Equal Power Mode (b) Power optimized mode

\section{CONCLUSIONS}

Achieving the higher spectrum efficiency is the primary goal of cognitive radio technology. With this motto, we have implemented the non-orthogonal multiple access (NOMA) in cognitive radio networks to support multiple simultaneous transmissions. More particularly, we have proposed dynamic QoS based power allocation algorithm for both uplink and downlink scenarios. The proposed optimization outperforms conventional NOMA in cognitive radio networks in terms of overall system throughput even satisfying the QOS requirements of users of individual network. Furthermore, mathematical expressions are derived for system outage probability considering both downlink and uplink scenarios. Simulations are carried out for both ordered and unordered NOMA for different values of SNR. It can be seen from results that; outage probability of PU can be is greatly reduced by adopting QoS based power allocation technique without affecting the secondary user performance. The system outage probability reduces as SNR increases up to certain value, but at high SNR system outage probability is dependent only on QoS constraints and power allocation coefficient. The simulation results of this work show that, CR-NOMA can achieve higher throughput and simultaneously prevents primary users from outage through optimized power allocation technique.

\section{REFERENCES}

[1] Lee, S., Zhang, R. (2015). Cognitive wireless powered network: Spectrum sharing models and throughput maximization. IEEE Transactions on Cognitive Communications and Networking, 1(3): 335-346. https://doi.org/10.1109/TCCN.2015.2508028

[2] Islam, S.R., Avazov, N., Dobre, O.A., Kwak, K.S. (2016). Power-domain non-orthogonal multiple access (NOMA) in 5G systems: Potentials and challenges. IEEE Communications Surveys \& Tutorials, 19(2): 721-742.

[3] Shirvanimoghaddam, M., Dohler, M., Johnson, S.J. (2017). Massive non-orthogonal multiple access for cellular IoT: Potentials and limitations. IEEE Communications Magazine, 55(9): 55-61. https://doi.org/10.1109/MCOM.2017.1600618

[4] Mohammadi, M., Shi, X., Chalise, B.K., Ding, Z., Suraweera, H.A., Zhong, C., Thompson, J.S. (2019). Full-duplex non-orthogonal multiple access for next generation wireless systems. IEEE Communications Magazine, $\quad 57(5)$ :

110-116. https://doi.org/10.1109/MCOM.2019.1800578

[5] Pandit, S., Singh, G. (2017). An overview of spectrum sharing techniques in cognitive radio communication system. Wireless Networks, 23(2): 497-518. https://doi.org/10.1007/s11276-015-1171-1 
[6] Zhang, D., Zhou, Z., Xu, C., Zhang, Y., Rodriguez, J., Sato, T. (2017). Capacity analysis of NOMA with mmWave massive MIMO systems. IEEE Journal on Selected Areas in Communications, 35(7): 1606-1618. https://doi.org/10.1109/JSAC.2017.2699059

[7] Le, C.B., Do, D.T., Voznak, M. (2019). Wirelesspowered cooperative MIMO NOMA networks: Design and performance improvement for cell-edge users. Electronics, $8(3)$ : 328. https://doi.org/10.3390/electronics8030328

[8] Dai, L., Wang, B., Ding, Z., Wang, Z., Chen, S., Hanzo, L. (2018). A survey of non-orthogonal multiple access for 5G. IEEE Communications Surveys \& Tutorials, 20(3): 2294-2323 https://doi.org/10.1109/COMST.2018.2835558

[9] Ding, Z., Zhu, Y., Chen, Y. (2019). Conclusions and future research directions for NOMA. In Multiple Access Techniques for 5G Wireless Networks and Beyond, pp. 669-677. https://doi.org/10.1007/978-3-319-92090-0_21

[10] Islam, S.M., Zeng, M., Dobre, O.A. (2017). NOMA in $5 \mathrm{G}$ systems: Exciting possibilities for enhancing spectral efficiency. arXiv preprint arXiv:1706.08215. https://arxiv.org/abs/1706.08215v1

[11] Wei, Z., Yang, L., Ng, D.W.K., Yuan, J., Hanzo, L. (2019). On the performance gain of NOMA over OMA in uplink communication systems. IEEE Transactions on Communications, $\quad 68(1)$ : 536-568. https://doi.org/10.1109/TCOMM.2019.2948343

[12] Tang, J., Luo, J., Liu, M., So, D.K., Alsusa, E., Chen, G., Wong, K., Chambers, J.A. (2019). Energy efficiency optimization for NOMA with SWIPT. IEEE Journal of Selected Topics in Signal Processing, 13(3): 452-466. https://doi.org/10.1109/JSTSP.2019.2898114

[13] Zhang, H., Feng, M., Long, K., Karagiannidis, G.K., Leung, V.C., Poor, H.V. (2019). Energy efficient resource management in SWIPT enabled heterogeneous networks with NOMA. IEEE Transactions on Wireless Communications, $\quad$ 19(2): 835-845. https://doi.org/10.1109/TWC.2019.2948874

[14] Thakur, P., Kumar, A., Pandit, S., Singh, G., Satashia, S.N. (2019). Frameworks of non-orthogonal multiple access techniques in cognitive radio communication systems. China Communications, 16(6): 129-149. https://doi.org/10.23919/JCC.2019.06.011

[15] Zhou, F., Wu, Y., Liang, Y.C., Li, Z., Wang, Y., Wong, K.K. (2018). State of the art, taxonomy, and open issues on cognitive radio networks with NOMA. IEEE Wireless Communications, 25(2): 100-108 https://doi.org/10.1109/MWC.2018.1700113

[16] Liu, C.H., Liang, D.C. (2018). Heterogeneous networks with power-domain NOMA: Coverage, throughput, and power allocation analysis. IEEE Transactions on Wireless Communications, 17(5): 3524-3539. https://doi.org/10.1109/TWC.2018.2816923

[17] Vaezi, M., Schober, R., Ding, Z., Poor, H.V. (2019). Non-orthogonal multiple access: Common myths and critical questions. IEEE Wireless Communications, 26(5): $174-180$ https://doi.org/10.1109/MWC.2019.1800598

[18] Cichoń, K., Kliks, A., Bogucka, H. (2016). Energyefficient cooperative spectrum sensing: A survey. IEEE
Communications Surveys \& Tutorials, 18(3): 1861-1886 https://doi.org/10.1109/COMST.2016.2553178

[19] Anandakumar, H., Umamaheswari, K. (2017). An efficient optimized handover in cognitive radio networks using cooperative spectrum sensing. Intelligent Automation \& Soft Computing, 1-8. https://doi.org/10.1080/10798587.2017.1364931

[20] Agarwal, A., Jagannatham, A.K. (2018). Performance analysis for non-orthogonal multiple access (NOMA)based two-way relay communication. IET Communications, $\quad$ 13(4): 363-370. https://doi.org/10.1049/iet-com.2018.5641

[21] Bishnu, A., Bhatia, V. (2019). Cognitive radio networks: IEEE 802.22 standards. Sensing Techniques for Next Generation Cognitive Radio Networks. IGI Global, pp. 27-50. https://doi.org/10.4018/978-1-5225-5354$0 . \operatorname{ch} 002$

\section{NOMENCLATURE}

$\begin{array}{ll}\mathrm{x}_{\mathrm{p}} & \begin{array}{l}\text { Primary user symbol } \\ \mathrm{X}_{\mathrm{s}}\end{array} \\ \rho_{\mathrm{s}} & \text { Secondary user symbol } \\ \mathrm{h}_{\mathrm{i}} & \text { Total transmit power } \mathrm{SNR}, \mathrm{dB} \\ \mathrm{B} & \text { Channel power gain of the user i } \\ \mathrm{W}_{\mathrm{i}} & \text { Bandwidth, Hz } \\ & \text { Noise power (AWGN) in downlink scenario, } \\ \mathrm{R} & \mathrm{dB}\end{array}$

\section{Greek symbols}

$\alpha \quad$ Power allocation coefficient.

$\beta \quad$ Rayleigh fading channel coefficient.

$\gamma \quad$ Signal to noise ratio, $\mathrm{dB}$

$\delta \quad \delta^{2}=\mathrm{E}\{\beta\}$ mean

\section{Abbreviations}

$\begin{array}{ll}\text { PU } & \text { Primary User } \\ \text { SU } & \text { Secondary User } \\ \text { CR } & \text { Cognitive Radio } \\ \text { NOMA } & \text { Non -Orthogonal Multiple Access } \\ \text { SC } & \text { Superposition Coding } \\ \text { SIC } & \text { Successive Interference Cancellation } \\ \text { FD } & \text { Full Duplex } \\ \text { MIMO } & \text { Multiple-Input, Multiple-Output } \\ \text { QoS } & \text { Quality of Service }\end{array}$

